\title{
Numerical simulation and prediction of groundwater environmental contamination in a coastal polyurethane plant based on GMS
}

\author{
Haodong Gao ${ }^{1, a}$, Shanshan Zhang ${ }^{2}$, Ruifeng Zhao ${ }^{2}$, Donghao $\mathrm{Xu}^{2}$, and Liangchao Zhu ${ }^{2}$ \\ ${ }^{1}$ Yantai Institute, China Agriculture University, 264670 Yantai, Shandong, China \\ ${ }^{2}$ Shandong Haiyue Environmental Technology Co., Ltd., 264670 Yantai, Shandong, China
}

\begin{abstract}
GMS was used to simulate the groundwater pollution status during the operation period of a coastal polyurethane factory. Based on the geological and hydraulic data, the groundwater flow field model and solute transport model were established using the MODFLOW and MT3DMS module respectively by which the potential area and concentration of pollutant were predicted and the extent of groundwater pollution in the study area was evaluated. The simulation and prediction results showed that under abnormal conditions, the groundwater environment would be affected. As the characteristic pollutants, COD and ethylene glycol mainly migrated along the direction of water flow and exceeded the standard for a certain period of time. The migration distance and pollution range of contaminants expanded with time, and the peak concentration decreased with the increase of diffusion distance. After 50 years, the potential pollution area of COD and ethylene glycol would reach $225651.2 \mathrm{~m}^{2}$ and $210947.5 \mathrm{~m}^{2}$ respectively. During the migration process, the concentration of pollutants in the groundwater gradually recovered to the background value, and the over-standard area was reduced to zero. The comprehensive prediction model and risk assessment method of this study can help to formulate practical prevention and control measures for the protection of groundwater environment.
\end{abstract}

\section{Introduction}

As an indispensable water resource, groundwater meets various water demands in social and economic development and maintains a wide diversity of ecosystem functions and services. However, on a global scale, due to the highly centralized development of industry and agriculture, a large number of pollutants are infiltrated into groundwater through landfill, sewage discharge, waste accumulation, fertilization, organic pollutants and oil leakage, which pose a serious threat to groundwater resources[1]. The risk assessment of groundwater pollution has important practical significance for coordinating the relationship between resource utilization and ecological environment[2]. Due to the great difficulty and high economic cost of the realtime monitoring of groundwater pollutants, groundwater simulation software has been developed, which can be used not only for groundwater pollution assessment, optimal management of groundwater, but also for solving the safety problems in resource exploitation. GMS (Groundwater Modeling System) is a visualized groundwater numerical simulation software based on finite difference method, integrating various modules such as MODFLOW and MT3D. GMS has the advantages of friendly operation interface, multivariable modules, all-around functions, wide application range and intuitive modeling process. It has been widely

\footnotetext{
a Corresponding author: oceangog@163.com
}

applied to establish water flow model and solute transport model for groundwater resource evaluation, pollution prediction, and groundwater management [3].

With the rapid development of chemical, pharmaceutical, pesticide and other industries in the coastal areas, the pollution problems caused by overexploitation have become increasingly prominent, especially for the pollution of groundwater environment, which is concealed, hysteretic and difficult to remove. In addition, the hydraulic connection between groundwater, surface water and seawater in the coastal area is close and complicated. However, studies on groundwater environment evaluation in the coastal area are very limited presently, which makes it a difficult point in the project environmental assessment. The studied coastal polyurethane factory is equipped with sewage treatment and raw material storage equipments, which constitutes a potential source of pollution. The large amount of "three wastes" with complex components will cause serious harm to the groundwater environment in case of leakage. GMS was used to simulate the groundwater pollution situation during the operation period of the polyurethane plant. The groundwater flow field model established by MODFLOW module was combined with the solute transport model established by MT3DMS module to predict the potential area and concentration of pollutant migration. Finally, the scope and extent of groundwater 
pollution in the plant area were evaluated, which provided a scientific basis for groundwater quality prediction and pollution control.

\section{Hydrogeological overview}

According to the engineering survey report and drilling data, the lithologic combination of the water-bearing medium in the evaluation area and the occurrence characteristics of groundwater were studied. The study area was a floodplain zone characterized by porosity aquifer in quaternary period, and the water-bearing medium was mainly composed of fine sand, medium fine sand, coarse sand and gravel. The thick aquifer with large pores provided ample space for groundwater accumulation. The hydrochemical types of groundwater were mainly $\mathrm{HCO}_{3} \cdot \mathrm{SO}_{4}-\mathrm{Ca}$ and $\mathrm{Cl} \cdot \mathrm{SO}_{4}-\mathrm{Ca} \bullet \mathrm{Mg}$. The total dissolved solids were at $471 \sim 892 \mathrm{mg} / \mathrm{L}$ and the well water inflow was $500 \sim 1000 \mathrm{~m}^{3} / \mathrm{d}$. The vertical infiltration of atmospheric precipitation and the recharge of surface water were the main sources of groundwater supply. Groundwater was consumed mainly by underground runoff, artificially exploitation and soil evaporation. The groundwater flow direction was from south to north, and the runoff was strictly controlled by the terrain.

\section{Methodology}

The hydrogeological conceptual model was established based on the analysis of the groundwater flow characteristics, aquifer system structure and boundary conditions according to the literature and on-site hydrogeological environment survey results in the study area; The groundwater flow model was constructed by spatial dispersion, assignment of hydrogeological parameters, and verification of groundwater flow field; Reasonable pollution factors and groundwater leakage scenarios were selected and designed according to the characteristics of pollution sources. The groundwater flow model was coupled with the pollutant transport model to simulate and predict the migration process of pollutants in the groundwater of the study area. Finally, reasonable water quality standards were selected to evaluate the simulation results [4].

\subsection{Hydrogeological model generalization}

The main aquifer group in the study area was porosity aquifer in quaternary period. The aquifer had good connectivity and a uniform runoff field. Groundwater was dominated by horizontal movement with a runoff from south to north. The upper boundary of the aquifer was the ground through which the aquifer system produced vertical water and solute exchange with atmospheric precipitation, surface water, and the like. The lower boundary contained a weakly permeable silty clay layer of about $5 \mathrm{~m}$, which blocked the hydraulic connection of different aquifers, and it was defined as the confining boundary. The north side of the simulation area was close to the Yellow Sea, and the east side was a river. According to long-term observation data, the groundwater level changed little, so they were regarded as the boundary of the fixed head. The north boundary of the area was basically the boundary between the Quaternary and the mountain bedrock, while the periphery area was the exposed area of the bedrock. The perimeter of the simulated area was defined as the flow boundary because of the lateral recharge from the bedrock fissure water to the Quaternary aquifer in the area. In the contaminant transport model, all boundaries were constant concentration boundaries. From January 2014 to May 2016, the groundwater level of the area was observed to be $24.65 \sim 25.32 \mathrm{~m}$ with a variable amplitude of $0.67 \mathrm{~m}$. Affected by atmospheric precipitation, the dynamic change of groundwater was small and the water level was basically stable. Through the comprehensive analysis of the above hydrogeological conditions, a hydrogeological conceptual model was established, and the groundwater aquifer system in the study area was generalized into a heterogeneous isotropic twodimensional steady flow problem.

\subsection{Numerical simulation of groundwater flow}

The mathematical model of groundwater flow field was established based on the hydrogeological conceptual model, and was solved by using the MODFLOW module in GMS software.

\subsubsection{Grid subdivision}

MODFLOW is a three-dimensional finite difference numerical simulation model specifically designed for groundwater flow in porous media. The simulation model covered a total area of about $5.4 \mathrm{~km}^{2}$, and was divided into 29 rows and 25 columns of rectangular grids with $100 \mathrm{~m} \times 100 \mathrm{~m}$ equidistant orthogonal elements on the plane, of which 523 were effective units. The areal discretization was shown in Figure 1.

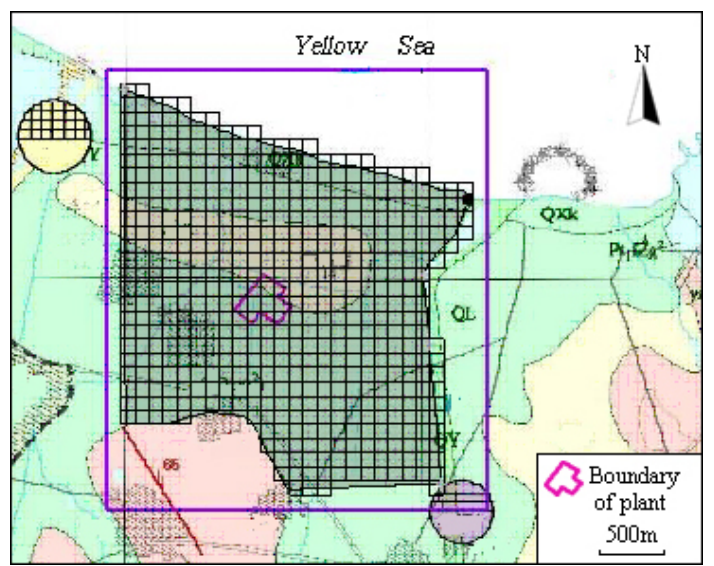

Figure 1. Areal discretization of the MODFLOW.

\subsubsection{Model parameters}

The hydrogeological parameters and solute transport parameters used in the calculation model were obtained based on the preliminary exploration drilling data, the various hydrogeological test data in the study area and the empirical values in the guidelines. Among them, the 
permeability coefficient was $10.0(\mathrm{~m} / \mathrm{d})$, the specific yield was 0.2 , the effective porosity was 0.3 , and the dispersion coefficient was $0.5\left(\mathrm{~m}^{2} / \mathrm{d}\right)$. The permeability coefficient of the aquifer was not partitioned in the horizontal direction because of the continuous and stable distribution of rock formations and the same aquifer type in the simulated area.

\subsubsection{Model identification and verification}

The groundwater flow field model was identified by the measured plane flow field in January 2016. On the basis of optimizing the parameters of the aquifer, the fitting of the flow field was completed. Analysis of the simulated flow field (Figure 2) showed that the groundwater flowed from south to north, which was basically consistent with the actual groundwater flow characteristics. The differences between the actual observed water levels and the model calculated water levels were all within $0.8 \mathrm{~m}$, representing a satisfactory fitting result. The water balance calculation results showed that there was a close hydraulic connection between groundwater and surface water. In summary, the model basically reflected the hydraulic characteristics of the groundwater system in the assessment area and could be used for the prediction and evaluation of groundwater environmental impact.

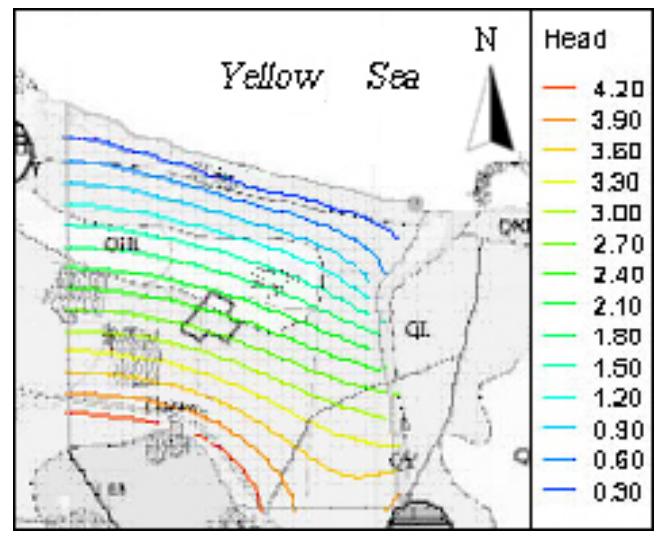

Figure 2. Simulated flow field of groundwater.

\subsection{Numerical simulation of groundwater solute transport}

The MT3DMS module in GMS is a three-dimensional solute transport model that simulates convection, dispersion, and chemical reactions in groundwater systems. Based on the identified groundwater flow field model, the solute (contaminant) migration model was established using the MT3DMS module to simulate and predict the migration characteristics and concentration change trends of pollutants during the operation period of the plant[5]. The range and boundary position of the solute transport model were consistent with the water flow model. According to the engineering characteristics of the evaluation area and the physical and chemical characteristics of the pollutants, in the principle of risk maximization, the adsorption, volatilization and biochemical reactions of pollutants in the aquifer were not considered in the groundwater pollution simulation process. The parameters in the model were conservatively considered. The simulation prediction was carried out according to the project service period of $50 \mathrm{a}$, and the contaminants migration situations after $100 \mathrm{~d}, 1000 \mathrm{~d}, 20 \mathrm{a}$ and $50 \mathrm{a}$ of pollution were predicted.

\section{Groundwater impact prediction and assessment}

This simulation predicted the migration process of pollutants in groundwater under accident conditions, and further analyzed the contaminant impact range, overstandard range and the concentration changes of pollution plumes. The spatial and temporal distribution of COD and ethylene glycol in the groundwater at 100d, $1000 \mathrm{~d}, 20 \mathrm{a}$ and $50 \mathrm{a}$ under abnormal conditions were shown as contamination plumes on the concentration contour map (Figure 3 and Figure 4).

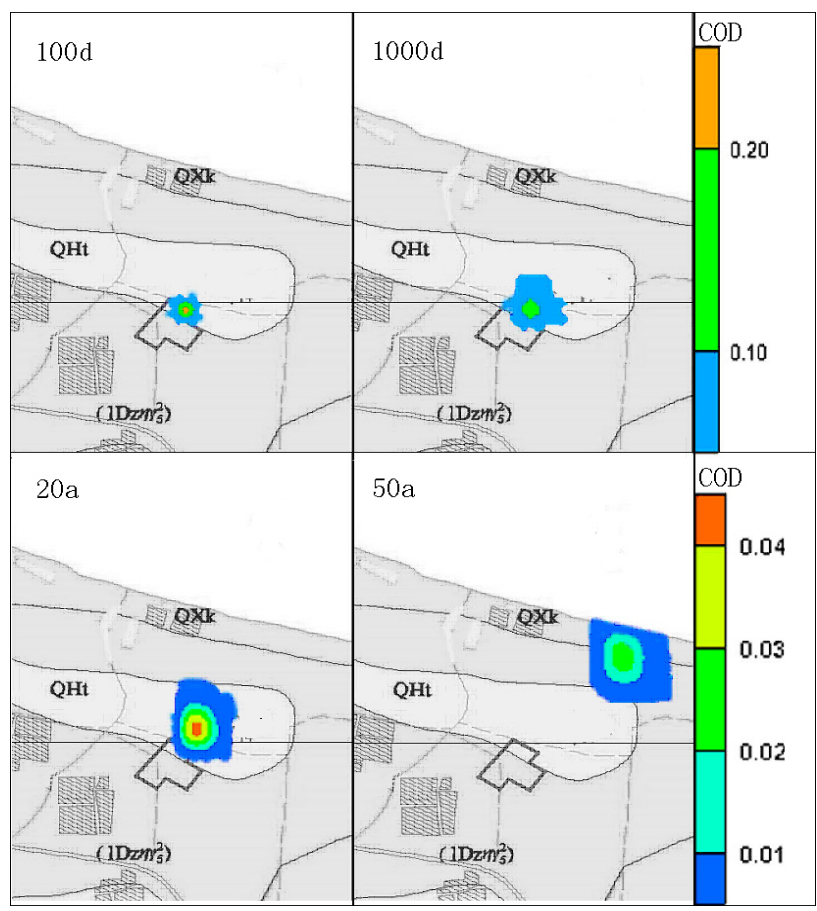

Figure 3. Spatial and temporal distribution of $\mathrm{COD}(\mathrm{mg} / \mathrm{L})$.

Figure. 3 showed that after the leakage of the sewage treatment station, the infiltration of sewage lead to an increase in the concentration of COD in the groundwater. The pollutants were mainly concentrated in the factory field with a maximum concentration of $3.26 \mathrm{mg} / \mathrm{L}$ at 100 days, and the COD on the north side of the field exceeded the standard. As time went by, the pollutants migrated with the groundwater flow field as a whole to the east-north direction downstream. The COD in the groundwater of the downstream areas underwent a process of concentration change that gradually increased, and then gradually decreased to near the background value. During the migration process, the peak concentration of COD was continuously reduced, and the pollution area continuously expanded. After 20 years, the pollutants migrated out of the field, and after 50 years, the peak concentration of COD in the area was about $0.03 \mathrm{mg} / \mathrm{L}$. 
Figure 4 showed that the highest concentration of ethylene glycol in the nearby groundwater within $100 \mathrm{~d}$ would reach $3.0 \mathrm{mg} / \mathrm{L}$ due to the leakage of storage tank. After the occurrence of the leak, the distribution range of ethylene glycol in the groundwater gradually increased with time, the highest concentration continuously decreased, and the peak concentration point continuously migrated outward. After 3 years, the pollutants moved outside the factory area, and after about 30 years, the pollutants were completely removed from the field. The peak concentration of ethylene glycol in groundwater decreased to about $0.3 \mathrm{mg} / \mathrm{L}$ in about 50 years.

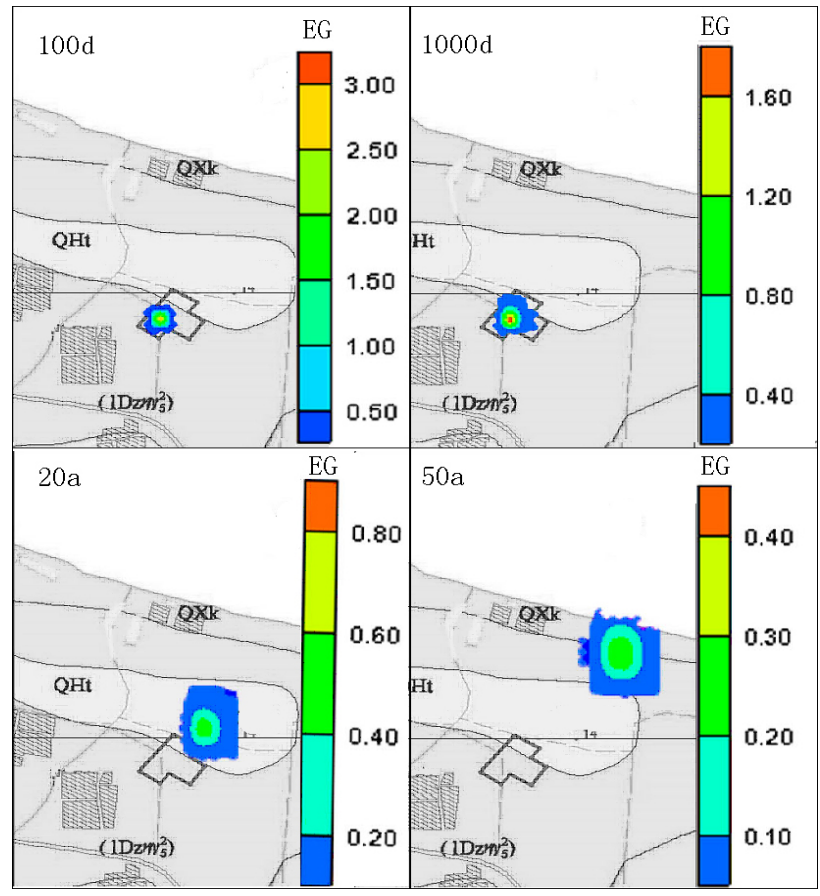

Figure 4. Spatial and temporal distribution of $\mathrm{EG}(\mathrm{mg} / \mathrm{L})$.

The simulation results showed that the infiltration of sewage would affect the aquifer. With the passage of time, the central concentration of the pollutants would gradually increase and exceed the standard within a certain range. The pollutants spread out in the direction of water flow, resulting in an increase in the migration distance and pollution range. At the same time, due to the effect of dispersion and convection, the central concentration of pollutants gradually decreased with the increase of the diffusion distance, and the over-standard area gradually shrank to zero, which indicated the disappearance of pollution influence on the aquifer. The detail migration characteristics of COD and ethylene glycol were listed in table 1 and table 2 .

Table 1. Migration characteristics of COD.

\begin{tabular}{|c|c|c|}
\hline $\begin{array}{c}\text { Prediction } \\
\text { time }\end{array}$ & $\begin{array}{c}\text { Migration } \\
\text { distance/m }\end{array}$ & $\begin{array}{c}\text { Influence } \\
\text { area/m }\end{array}$ \\
\hline $100 \mathrm{~d}$ & 211.3 & 38410.1 \\
\hline $1000 \mathrm{~d}$ & 265.0 & 100067.8 \\
\hline $20 \mathrm{a}$ & 453.2 & 176385.3 \\
\hline $50 \mathrm{a}$ & 1044.3 & 225651.2 \\
\hline
\end{tabular}

Table 2. Migration characteristics of ethylene glycol.

\begin{tabular}{|c|c|c|}
\hline $\begin{array}{c}\text { Prediction } \\
\text { time }\end{array}$ & $\begin{array}{c}\text { Migration } \\
\text { distance/m }\end{array}$ & $\begin{array}{c}\text { Influence } \\
\text { area/ } \mathrm{m}^{2}\end{array}$ \\
\hline $100 \mathrm{~d}$ & 87.3 & 33558.9 \\
\hline $1000 \mathrm{~d}$ & 91.0 & 55317.2 \\
\hline $20 \mathrm{a}$ & 397.8 & 152231.6 \\
\hline $50 \mathrm{a}$ & 1029.3 & 210947.5 \\
\hline
\end{tabular}

\section{Conclusions}

Based on the literature collection, data analysis and onsite hydrogeological environment investigation, reasonable simulation range was determined and the corresponding groundwater numerical model was constructed in this study. The model was identified and verified by observed water level and groundwater movement characteristics in the study area. The corrected model could truly reflect the actual hydrogeological conditions in the study area. According to the characteristics of the project in the evaluation area, a reasonable pollutant leakage scenario was designed, using COD and ethylene glycol as the characteristic pollutants. The groundwater flow numerical model was coupled with the pollutant migration equation to obtain the groundwater solute transport model, which was used to analyze the effects of pollutants on groundwater under abnormal conditions and predict the temporal and spatial migration of pollutants. The simulation and prediction results showed that under abnormal conditions, the groundwater environment in the accident area would be affected by pollution. COD and ethylene glycol mainly migrated along the direction of water flow and exceeded the standard for a certain period of time. The migration distance and pollution range of contaminants expanded with time, and the peak concentration decreased with the increase of diffusion distance. During the migration and diffusion process, the concentration of pollutants in the groundwater gradually recovered to the background value, and the over-standard area was reduced to zero. The comprehensive prediction model and risk assessment method of this study can help to formulate practical prevention and control measures, and have important positive significance for the protection of groundwater environment in polyurethane factories in coastal areas.

\section{References}

1. M. Stuart, D. Lapworth, E. Crane, A. Hart, Sci. Total Environ. 416, 1 (2012).

2. M. Shan, S. Zhang, Appl. Mech. Mater. 174, 3387 (2012) .

3. L.K. Lautz, D.I. Siege, Adv. Water. Resour. 29, 1618(2006).

4. Y. Wang, K.N. Zhang, Y.G. Chen, X.Z. Zhou, J. Cent. South. Univ. T. 20, 11 (2013). 
5. F.M. Liu, S.P. Yi, H.Y. Ma, J.Y. Huang, Y.K. Tang, J.B. Qin, W.H. Zhou, ENVIRON. SCI. POLLUT. R. 4, 1 (2017). 